\title{
Relativistic contributions to single and double core electron ionization energies of noble gases
}

\author{
J Niskanen, Patrick Norman, H Aksela and H Agren
}

\section{Linköping University Post Print}

N.B.: When citing this work, cite the original article.

Original Publication:

J Niskanen, Patrick Norman, H Aksela and H Agren, Relativistic contributions to single and double core electron ionization energies of noble gases, 2011, Journal of Chemical Physics, (135), 5, 054310.

http://dx.doi.org/10.1063/1.3621833

Copyright: American Institute of Physics (AIP)

http://www.aip.org/

Postprint available at: Linköping University Electronic Press

http://urn.kb.se/resolve?urn=urn:nbn:se:liu:diva-70332 


\title{
Relativistic contributions to single and double core electron ionization energies of noble gases
}

\author{
J. Niskanen, ${ }^{1,2, a)}$ P. Norman, ${ }^{3}$ H. Aksela, ${ }^{1}$ and H. Ågren ${ }^{2}$ \\ ${ }^{1}$ Department of Physics, University of Oulu, Box 3000, 90014 Oulu, Finland \\ ${ }^{2}$ Department of Theoretical Chemistry, School of Biotechnology, Royal Institute of Technology, \\ SE-106 91 Stockholm, Sweden \\ ${ }^{3}$ Linköping University, SE-581 83 Linköping, Sweden
}

(Received 23 November 2010; accepted 14 July 2011; published online 5 August 2011)

\begin{abstract}
We have performed relativistic calculations of single and double core $1 s$ hole states of the noble gas atoms in order to explore the relativistic corrections and their additivity to the ionization potentials. Our study unravels the interplay of progression of relaxation, dominating in the single and double ionization potentials of the light elements, versus relativistic one-electron effects and quantum electrodynamic effects, which dominate toward the heavy end. The degree of direct relative additivity of the relativistic corrections for the single electron ionization potentials to the double electron ionization potentials is found to gradually improve toward the heavy elements. The Dirac-Coulomb Hamiltonian is found to predict a scaling ratio of $\sim 4$ for the relaxation induced relativistic energies between double and single ionization. Z-scaling of the computed quantities were obtained by fitting to power law. The effects of nuclear size and form were also investigated and found to be small. The results indicate that accurate predictions of double core hole ionization potentials can now be made for elements across the full periodic table. () 2011 American Institute of Physics. [doi:10.1063/1.3621833]
\end{abstract}

\section{INTRODUCTION}

Modern experimental development has enabled studies of new processes and states of matter containing hollow atoms and multiply ionized states. Efficient multielectron detection time-of-flight (TOF) spectrometry ${ }^{1}$ and related photoelectronphotoelectron coincidence (TOF-PEPECO) spectroscopy ${ }^{2}$ have made it possible to study multiple ionization processes induced by single photons from synchrotron sources. Recently inaugurated, free electron laser (FEL) facilities offer sources of very intense radiation, capable of inducing sequential and direct multiphoton-multielectron processes. Such efforts, now taking place at, e.g., the Linac Coherent Light Source (LCLS) show the promise to measure the kinetic energies of two core photoelectrons generated via sequential absorption of two or more photons from a short x-ray pulse. ${ }^{3-5}$

Double photoionization by a single photon can be achieved at traditional synchrotron radiation sources and in TOF-PEPECO experiments. The dynamics of single photon double photoionization can be divided to a few interfering first order amplitudes arising from initial state electron correlation, electron knock-out, and shake-off processes. ${ }^{6}$ The dynamics of the one-photon double core photoionization has also gained interest recently ${ }^{7,8}$ and among other interesting features, the one-photon double ionization cross section has revealed to be a sensitive probe of initial and the final state electron correlation. The spearhead of current experimental efforts utilizes the potential of the recently opened x-ray FELs to produce photon energies sufficient to give rise to one-

\footnotetext{
a) Author to whom correspondence should be addresses. Electronic mail:
} johannes.niskanen@oulu.fi. photon deep core photoionization and field strengths capable to induce multiphoton processes. The latter can be divided in direct, nonsequential ionization (NSI), and sequential ionization (SI) based on the physical process of ionization. ${ }^{9,10}$ In a NSI multiphoton process, two or more photons are annihilated from the radiation field simultaneously with the requirement of conservation of the total energy of the system, while in a sequential process, photons are absorbed one by one, each step fulfilling the energy conservation principle. The SI processes are more limited by energy as for every step of the ionization process, an intermediate electronic state must be accessible. The sequential ionization has been found to be much more effective mechanism in the production of hollow $\mathrm{Ne}$ atoms than correlation driven one-photon double ionization. ${ }^{4}$ The complete understanding of the dynamics of these processes, however, still offers a considerable challenge.

The experimental endeavours in the area have spurred concomitant theoretical efforts, which in fact often have preceded the actual measurements. Recent studies have applied different multiconfiguration self-consistent field, perturbation theory, and density functional theory approaches to explore the nature of double core hole states. Calculations have indicated several interesting particularities with respect to the contribution of relaxation, electron correlation, Coulomb repulsion, and exchange. For instance, the residual (dynamical) relaxation between single and double ionization is very large for the two core electron ionization at the same site, while it is comparably small, and can even be negative, when the two electrons are emitted from different sites of the molecules. ${ }^{11}$ These contributions are both two-element specific and structurally dependent, amounting to a sizeable chemical shift that 
is very specific compared to the common case of single core electron ionization.

So far, experiments of the core hole states produced by double photoionization have been rather limited. The Ne $1 \mathrm{~s}$ double ionization potential (DIP) was early derived experimentally from a hypersatellite in the $\mathrm{x}$-ray emission spectrum of Neon by Ågren et al., ${ }^{12}$ and more recently by Pelicon and co-workers, ${ }^{13}$ and by Southworth et al. ${ }^{14}$ measuring the KKKLL hypersatellite Auger spectra. The $1 s$ double ionization of silver has also been reported. ${ }^{15}$ The single core ionization potentials (IPs) for the whole studied series from $\mathrm{Ne}$ to $\mathrm{Rn}$ can be found from the spectral line data re-analysis by Bearden et al. ${ }^{16}$. For $\mathrm{Ne}$, a more recent experiment has been reported by Pettersson and co-workers. ${ }^{17}$ The studies of hollow atoms have been reviewed by Winter and Aumayr, ${ }^{18}$ while the competition between decay channels of the double core hole states for elements with $Z \leq 36$ has also been studied by Chen. ${ }^{19}$

The developed new $x$-ray FELs promise photon fluxes and energies suitable for multiple sequential and direct core ionization of atoms and molecules. For example, the currently operating AMO instrument at LCLS covers the photon energy range from 480 to $2000 \mathrm{eV}$ (Ref. 20) with first harmonics photon energies reaching up to some $10 \mathrm{keV}$. After planned upgrades, photon energies up to $25 \mathrm{keV}$ may be reached. ${ }^{20}$ Even though it is evident that sequential double $1 s$ core ionization of high- $Z$ elements cannot be studied by the current experimental setups, future experiments can be expected to extend toward elements with higher $Z$ value. Meanwhile, computational studies offer the possibilities to unravel trends for the full range of stable elements in the periodic table.

In order to forego coming experiments and aid our understanding further on the formation of such hollow states we undertake in the present work a study of the relativistic contributions to double core electron ionization for the noble gas series from Neon to Radon using the 4-component DiracHartree-Fock method. We explore, in particular, the additivity of relativistic effects between single and double ionization and the coupling between relaxation and relativistic contributions with respect to nuclear charge and the nuclear charge distribution. The $Z$-scaling of the computational results is also discussed. For this purpose, we present calculations for $1 s^{-1}$ and $1 s^{-2}$ ionization energies performed for the noble gas series from Neon to Radon to cover the Z-range of stable elements in the periodic table.

\section{COMPUTATIONAL DETAILS}

The core ionization potentials and double ionization potentials have been determined for the noble gases (including from $\mathrm{Ne}$ to $\mathrm{Rn}$ ) by means of the $\triangle \mathrm{SCF}$ method at the Hartree-Fock level of theory and by employment of the nonrelativistic (NR) Lévy-Leblond (LL) Hamiltonian ${ }^{21}$ as well as the relativistic Dirac-Coulomb (DC), Dirac-Coulomb-Gaunt (DCG), and Dirac-Coulomb-Breit (DCB) Hamiltonians. All SCF calculations have been carried out by use of either the DIRAC program, ${ }^{22}$ in which case results are obtained for the LL, DC, and DCG zeroth-order Hamiltonians, or the GRASP (Ref. 23) program, in which case results are obtained for the DC zeroth-order Hamiltonian with DCG and DCB cor- rections determined by means of the first-order perturbation theory and with use of the RATIP program package. ${ }^{24}$ The relativistic atomic structure theory is discussed in detail in Ref. 25.

The calculations using the DIRAC program are based on a finite atomic orbital basis consisting of scalar, real, Gaussian functions, and, for a given large-component basis set, the small component basis functions are obtained by the application of the condition of the restricted kinetic balance. For Ne and Ar, we adopted Dunning's augmented correlation consistent polarized core valence quintuple- $\zeta$ (aug-ccpCV5Z) basis set, ${ }^{26,27}$ and, for $\mathrm{Kr}, \mathrm{Xe}$, and $\mathrm{Rn}$, we adopted Dyall's quadruple- $\zeta$ basis set. ${ }^{28}$ To allow for full flexibility in the atomic orbital basis and thereby enabling an accurate description of relaxed orbitals for the ionized species, we decontracted the basis sets for all elements. As an estimate of the basis set convergence, we also determined DIPs with employment of the corresponding lower order decontracted basis sets, i.e., basis sets with exponents taken from Dunning's aug-cc-pCVQZ basis set for Ne and Ar and Dyall's triple- $\zeta$ basis set for $\mathrm{Kr}$, Xe, and $\mathrm{Rn} .{ }^{28,29}$ This comparison reveals a maximum discrepancy of $44 \mathrm{meV}$, occurring for the nonrelativistic DIP results of $\mathrm{Ne}$, and we, therefore, consider our presented results as accurate with respect to the issue of basis set convergence. We note that the electronic relaxation effects are largest for the double ionized states and the comparison of DIP results is, therefore, the most critical one. In addition, calculations using the GRASP program are based on tabulated radial wave functions that are to be considered as representing the atomic basis set limit and comparisons made against the GRASP benchmark data in Sec. III give further evidence for the quality of the chosen basis sets. Calculations using the DIRAC and GRASP programs adopt the Gaussian and Fermi charge distributions, respectively, as representations of nuclei. Unless specified differently, values for the nuclear parameters were obtained from Ref. 30.

\section{RESULTS}

The results of our calculations are presented in Table I. For comparison, experimental values, taken from literature, are also given. In the following paragraphs, we discuss features of each correction studied.

IPs of the noble gas series from Ne to Rn cover the energy range from approximately 870 to $100000 \mathrm{eV}$, while DIPs range from some 1800 to $200000 \mathrm{eV}$. As seen from Table I, the calculations for single IP with the DCG Hamiltonian show good agreement with the experimental IP values through the whole series. The calculated DIP for Ne is also quite close to the experimental value. It has been noted that the error limits for the IP values given in Ref. 16 are not absolute. For example, for $\mathrm{Ne}$, the value given in Ref. $16(866.9 \pm 0.3 \mathrm{eV}) \mathrm{de}-$ viates more than 10 times the given error limit from the value obtained from Ref. $17(870.21 \mathrm{eV})$. This may be due to the method of indirect evaluation of the IP from existing energy data. Such determination usually relies on a decay cascade from which a set of equations is set up to solve the core IP. In such a scheme the errors can be expected to accumulate for the heavy elements. 
TABLE I. Calculated $1 s$ single and double ionization potentials (IPs and DIPs, respectively) in eVs. Relativistic corrections are given after the nonrelativistic values. Details can be found in text.

\begin{tabular}{lrrrrr}
\hline \hline Hamiltonian/rel. corr. & $\mathrm{Ne}$ & $\mathrm{Ar}$ & $\mathrm{Kr}$ & $\mathrm{Xe}$ & $\mathrm{Rn}$ \\
\hline Single core ionization potentials & & & & & \\
NR & 868.63 & 3195.4 & 14101.3 & 33251.5 & 87805.4 \\
Dirac & +1.17 & +13.8 & +257.7 & +1435.4 & +11176.8 \\
Gaunt & -0.34 & -2.5 & -23.7 & -88.9 & -419.0 \\
Total & 869.46 & 3206.7 & 14335.3 & 34598.0 & 98563.2 \\
Exp. & $870.21^{\mathrm{a}}$ & $3202.9 \pm 0.3^{\mathrm{b}}$ & $14325.6 \pm 0.8^{\mathrm{b}}$ & $34561.4 \pm 1.1^{\mathrm{b}}$ & $98404 \pm 14.1^{\mathrm{b}}$ \\
Double core ionization potentials & & & & & \\
NR & 1860.26 & 6629.7 & 28704.4 & 67286.5 & 176897.3 \\
Dirac & +2.54 & +28.9 & +528.3 & +2919.5 & +22605.5 \\
Gaunt & -0.41 & -3.2 & -32.2 & -123.3 & -590.1 \\
Total & 1862.39 & 6655.4 & 29200.6 & 70082.7 & 198912.6 \\
Exp. & $1863^{\mathrm{c}}$ & $\ldots$ & $\ldots$ & $\ldots$ & $\ldots$ \\
\hline
\end{tabular}

${ }^{\mathrm{a}}$ Reference 17.

${ }^{\mathrm{b}}$ Reference 16 .

${ }^{\mathrm{c}}$ Reference 13

\section{A. One-electron relativistic effects}

The one-electron relativistic contributions in $\mathrm{IP}_{\mathrm{DC}}$ and DIP $_{D C}$ were obtained as the difference of (D)IPs from the DC-SCF method and the NR-SCF method. The values, denoted as corr ${ }_{\mathrm{DC}}^{\mathrm{IP}}$ and corr ${ }_{\mathrm{DC}}^{\mathrm{DIP}}$, are given in Table I denoted by "Dirac." The ratio corr ${ }_{\mathrm{DC}}^{\mathrm{DIP}} /$ corr $_{\mathrm{DC}}^{\mathrm{IP}}$ is depicted in Fig. 1, where we have also presented the values obtained in a frozen orbital approximation including the relativistic Gaunt two-electron corrections $\left(\operatorname{corr}_{\mathrm{DCG}}^{(D) \mathrm{IP}}\right)$. We note that a factor 2 for the ratio means direct additivity of the relativistic correction.

Starting from a couple of $\mathrm{eVs}$ for $\mathrm{Ne}$, the relativistic oneelectron energy contribution naturally increases toward the heavier end of the noble gas series being $\sim 11000 \mathrm{eV}$ and $\sim 23000 \mathrm{eV}$ for Rn IP and DIP, respectively. The additivity of the one-electron relativistic energy in the IP and DIP is

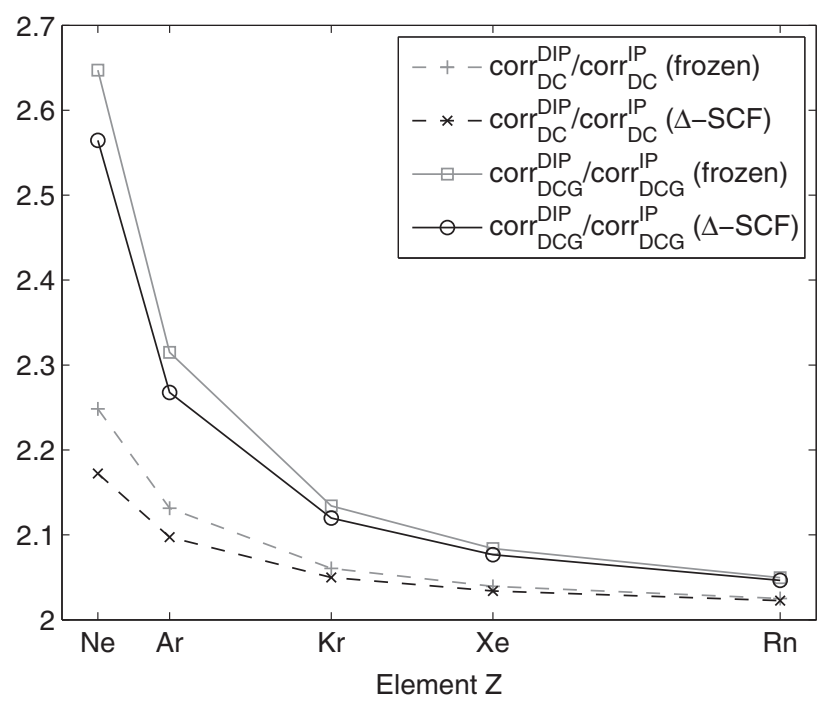

FIG. 1. The ratio of the relativistic corrections for the $1 s$ single ionization (corr ${ }^{\mathrm{IP}}$ ) and double ionization potentials (corr ${ }^{\mathrm{DIP}}$ ) for the noble gas series from Ne to Rn. Values were obtained using the Dirac-Coulomb (DC) and Dirac-Coulomb-Gaunt (DCG) Hamiltonians. The frozen-orbital values are also depicted. For details, see text. best fulfilled for the heavy end elements, as seen from Fig. 1. The ratio of the relativistic corrections to DIPs and IPs, thus, approaches systematically the value 2 when proceeding the series toward the heavy elements, as seen from Fig. 1. Even though the relativistic corrections to IPs and DIPs are small in absolute values for light elements, they differ relatively the most when the degree of ionization is increased from 1 to 2 . This may qualitatively be expected as low- $Z$ elements have fewer electrons for which relativistic effects are significant.

\section{B. Relaxation}

The relaxation energy contributions to the $1 s$ IPs and DIPs (relax ${ }^{\mathrm{IP}}$ and relax ${ }^{\mathrm{DIP}}$, respectively) were calculated at the NR, DC, and DCG levels as the difference between (D)IPs with the relaxed SCF orbitals and frozen ground state orbitals. The energies are given in Table II.

For IPs, the relaxation energy starts from $\sim-23 \mathrm{eV}$ for $\mathrm{Ne}$ and ends up around $-100 \mathrm{eV}$ for Rn. For DIPs, the relaxation energy begins from $-85 \mathrm{eV}$ for $\mathrm{Ne}$ and extends to around $-400 \mathrm{eV}$ for $\mathrm{Rn}$, thus roughly following the rule that the relaxation energy depends quadratically on the induced hole charge. ${ }^{31}$ The relative effect of relaxation is largest for $\mathrm{IP}_{\mathrm{DC}}$ and $\mathrm{DIP}_{\mathrm{DC}}$ of $\mathrm{Ne}$, affecting the second significant digit of the

TABLE II. Calculated relaxation energy contributions to the $1 s$ single and double ionization energies (relax ${ }^{\mathrm{IP}}$ and relax ${ }^{\mathrm{DIP}}$, respectively) in the nonrelativistic (NR), Dirac-Coulomb (DC), and Dirac-Coulomb-Gaunt (DCG) level (in $\mathrm{eV}$ ). Details can be found in text.

\begin{tabular}{lccccc}
\hline \hline & $\mathrm{Ne}$ & $\mathrm{Ar}$ & $\mathrm{Kr}$ & $\mathrm{Xe}$ & $\mathrm{Rn}$ \\
\hline $\operatorname{relax}_{\mathrm{NR}}^{\mathrm{IP}}$ & -23.16 & -32.16 & -52.98 & -64.83 & -85.09 \\
$\operatorname{relax}_{\mathrm{DC}}^{\mathrm{IP}}$ & -23.22 & -32.43 & -54.48 & -69.04 & -99.83 \\
$\operatorname{relax}_{\mathrm{DCG}}^{\mathrm{IP}}$ & -23.25 & -32.59 & -55.26 & -71.07 & -106.22 \\
$\operatorname{relax}_{\mathrm{NR}}^{\text {DIP }}$ & -85.81 & -122.55 & -206.17 & -253.79 & -334.94 \\
$\operatorname{relax}_{\mathrm{DC}}^{\mathrm{DIP}}$ & -86.03 & -123.61 & -211.95 & -270.15 & -392.12 \\
$\operatorname{relax}_{\mathrm{DCG}}^{\text {DIP }}$ & -86.12 & -124.08 & -214.39 & -276.63 & -412.76 \\
\hline \hline
\end{tabular}


TABLE III. Calculated relaxation induced relativistic energies in the $1 s$ single and double ionization potentials $\left(\Delta^{\mathrm{IP}}\right.$ and $\Delta^{\mathrm{DIP}}$, respectively). Values from both Dirac-Coulomb (DC) and Dirac-Coulomb-Gaunt (DCG) levels (in $\mathrm{eV}$ ) are given. Details can be found in text.

\begin{tabular}{lccccc}
\hline \hline & $\mathrm{Ne}$ & $\mathrm{Ar}$ & $\mathrm{Kr}$ & $\mathrm{Xe}$ & $\mathrm{Rn}$ \\
\hline$\Delta_{\mathrm{DC}}^{\mathrm{IP}}$ & -0.06 & -0.27 & -1.49 & -4.21 & -14.74 \\
$\Delta_{\mathrm{DCG}}^{\mathrm{IP}}$ & -0.09 & -0.43 & -2.27 & -6.24 & -21.13 \\
$\Delta_{\mathrm{DC}}^{\mathrm{DIP}}$ & -0.22 & -1.05 & -5.79 & -16.36 & -57.18 \\
$\Delta_{\text {DCG }}^{\text {DIP }}$ & -0.30 & -1.53 & -8.22 & -22.84 & -77.82 \\
\hline \hline
\end{tabular}

IP, while at the heavy end of the series, the effect of relaxation is seen in the third significant digit of the IP.

As observed in Table II, all three levels of theory give agreeing relaxation energies for the IP and DIP of Ne, while toward the heavy end of the noble gas series, the values begin to differ. The relaxation energies from the NR framework differ the most from the relativistic values for Rn. For all atoms, the DCG Hamiltonian gives the largest absolute values for relaxation energy. As seen in Table I and Table II, the relativistic one-electron contributions dominate for IPs and DIPs in the heavy end of the series, whereas the effect of relaxation dominates at the light end of the series.

The relativistic energy $\Delta$ induced by orbital relaxation was calculated from

$$
\Delta=\left(\mathrm{IP}_{\mathrm{DC}(\mathrm{G})}^{\text {relax }}-\mathrm{IP}_{\mathrm{NR}}^{\text {relax }}\right)-\left(\operatorname{IP}_{\mathrm{DC}(\mathrm{G})}^{\text {nonrelax }}-\mathrm{IP}_{\mathrm{NR}}^{\text {nonrelax }}\right) .
$$

The values for $\Delta^{\mathrm{IP}} \mathrm{S}$ and $\Delta^{\mathrm{DIP}} \mathrm{s}$ are given in Table III and depicted in Fig. 2. The relaxation induced relativistic contribution in (D)IPs shows monotonically increasing absolute values. Similarly, the DIP/IP ratio of the DC relaxation induced relativistic energies also shows a monotonically increasing trend, from 3.81 for Ne to 3.87 for $\mathrm{Rn}$. The corresponding DCG values evolve from 3.42 of $\mathrm{Ne}$ to 3.68 of $\mathrm{Rn}$. We also note that the absolute values of the relaxation induced rela-

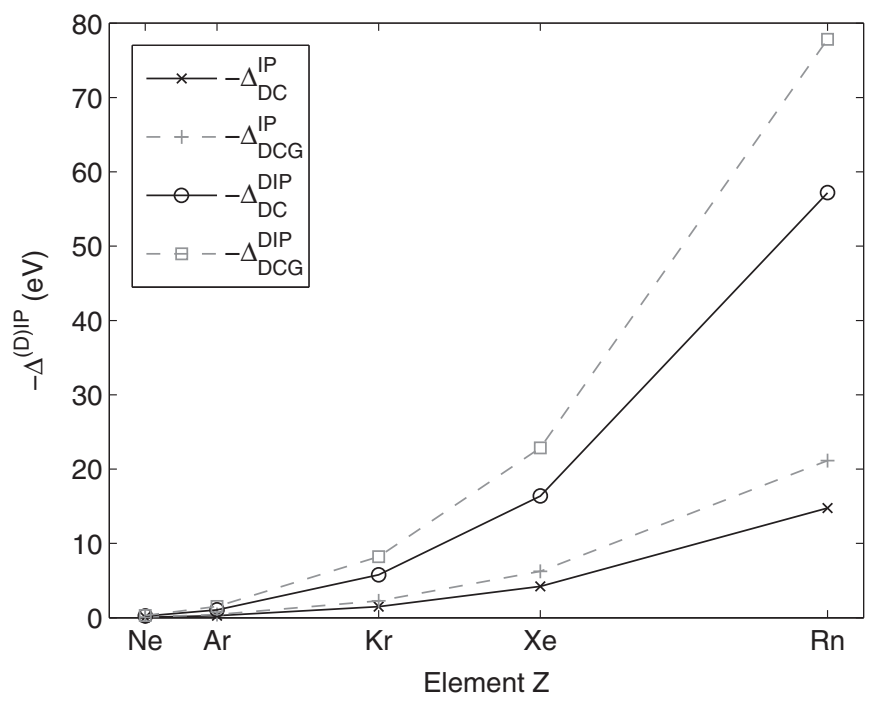

FIG. 2. Calculated relaxation induced relativistic energies (with opposite sign) in the $1 s$ single and double ionization potentials $\left(-\Delta^{\mathrm{IP}}\right.$ and $\left.-\Delta^{\mathrm{DIP}}\right)$. Values from both Dirac-Coulomb (DC) and Dirac-Coulomb-Gaunt (DCG) levels (in $\mathrm{eV}$ ) are shown. Details can be found in text.
TABLE IV. Calculated $1 s$ single and double ionization energies (in eV) with the Gaussian and Fermi nuclei. Dirac-Coulomb and Dirac-CoulombGaunt levels of theory were used. All values were obtained using the relaxed orbitals. Details can be found in text.

\begin{tabular}{lccccr}
\hline \hline & $\mathrm{Ne}$ & $\mathrm{Ar}$ & $\mathrm{Kr}$ & $\mathrm{Xe}$ & $\mathrm{Rn}$ \\
\hline IP $_{\text {DC }}$, Gaussian & 869.79 & 3209.2 & 14359 & 34687 & 98982 \\
IP $_{\text {DC }}$, Fermi & 869.79 & 3209.2 & 14359 & 34687 & 98973 \\
IP $_{\text {DCG }}$, Gaussian & 869.45 & 3206.7 & 14335 & 34598 & 98563 \\
IP $_{\text {DCG }}$, Fermi & 869.46 & 3206.7 & 14335 & 34599 & 98562 \\
DIP $_{\text {DC }}$, Gaussian & 1862.8 & 6658.6 & 29233 & 70206 & 199503 \\
DIP $_{\text {DC }}$, Fermi & 1862.8 & 6658.6 & 29233 & 70206 & 199485 \\
DIP $_{\text {DCG }}$, Gaussian & 1862.4 & 6655.4 & 29201 & 70083 & 198913 \\
DIP $_{\text {DCG }}$, Fermi & 1862.4 & 6655.4 & 29201 & 70084 & 198911 \\
\hline \hline
\end{tabular}

tivistic energies are larger in absolute values for DCG than for DC.

\section{Dependence on the nuclear model}

The dependence of IPs and DIPs on the used nuclear model was studied at the DC and DCG levels. The results are presented in Table IV, which show DC-SCF IPs and DIPs with a Gaussian (DIRAC code) nucleus accompanied by the values from DC-SCF calculation using Fermi nucleus (GRASP code). The values for the DCG Hamiltonian are presented similarly. The nuclear parameters were taken from Ref. 30.

As seen from the values of Table IV, there is no significant difference between the used nuclear model. Interestingly, $\mathrm{IP}_{\mathrm{DC}} \mathrm{s}$ for $\mathrm{Rn}$ differ by $9 \mathrm{eV}$ while the DCG values remain closely the same. For DIPs, similar observations are made. The given values also include the effect of finite basis set as the Fermi nucleus model was used in the GRASP calculation, where tabulated radial wave functions are being used. We find that the used finite Gaussian basis sets and nuclear charge distribution reproduce well the results of complete basis set limit and the more realistic Fermi nuclear charge distribution.

The effect of the used nuclear size was calculated using the Gaussian model for Rn by the DIRAC code. The nuclear radius with a root-mean-square $(\mathrm{rms}) r_{0}^{\mathrm{rms}}$ was scaled down to $75 \%$ of the rms radius (exponents $1.32423502 \times 10^{8}$ and $2.35419559 \times 10^{8}$ in a.u., respectively). The results of the calculation are given in Table V. The IP and DIP values obtained by the DC method are more dependent of the nuclear size than the values obtained by the NR method. In the radical change of the nuclear rms size, the maximal nuclear size effect $\left(34.93 \mathrm{eV}\right.$ for $\mathrm{IP}_{\mathrm{DC}}$ and $70.60 \mathrm{eV}$ for $\left.\mathrm{DIP}_{\mathrm{DCG}}\right)$ is found in the fourth significant digit of (D)IPs. Orbital relaxation has negligible effect in the difference.

\section{Relativistic electron-electron interaction corrections}

Depending on the gauge used for the electromagnetic field, the first relativistic electron-electron interaction term results in different forms. ${ }^{25}$ The Gaunt operator form is obtained in the long-wavelength limit of applying the Feynman 
TABLE V. Calculated $1 s$ single and double ionization energies (in eV) with two different nuclear radii and their difference. Values were obtained using relaxed orbitals. Details can be found in text.

\begin{tabular}{lrrrr}
\hline \hline & \multicolumn{1}{c}{$\mathrm{IP}_{\mathrm{NR}}$} & \multicolumn{1}{c}{$\mathrm{IP}_{\mathrm{DC}}$} & \multicolumn{1}{c}{$\mathrm{DIP}_{\mathrm{NR}}$} & \multicolumn{1}{c}{$\mathrm{DIP}_{\mathrm{DC}}$} \\
\hline$r_{0}^{\mathrm{rms}}$ & 87805.43 & 98982.18 & 176897.26 & 199502.73 \\
$0.75 \times r_{0}^{\mathrm{rms}}$ & 87810.11 & 99017.11 & 176906.70 & 199573.33 \\
Difference & 4.68 & 34.93 & 9.44 & 70.60 \\
\hline \hline
\end{tabular}

gauge whereas the long-wavelength limit in the Coulomb gauge results in the frequency-independent transverse Breit operator as correction to the Coulomb operator. To study the consistency of the two models for relativistic electronelectron interaction effects, we applied the Dirac-Coulomb, Dirac-Coulomb-Gaunt, and Dirac-Coulomb-(frequencyindependent transverse) Breit Hamiltonians using the GRASP package and the related RATIP package. The corrections are effectively the difference between (D)IP $\mathrm{DCG}_{\mathrm{B} / \mathrm{B}}$ and (D)IP $\mathrm{IC}_{\mathrm{DC}}$ and are given in Table VI. For comparison, the corresponding values for the Gaunt term obtained by the DIRAC package are also given.

Table VI shows that the transverse Breit and Gaunt corrections deviate increasingly toward the heavy end of the noble gas series. A smaller deviation is also seen between the Gaunt corrections obtained by different codes. The ratio of the Gaunt corrections in the DIP and IP (Gaunt $\left.{ }^{(\mathrm{D}) I P}\right)$ and the corresponding ratio for the transverse Breit correction $\left(\mathrm{Breit}^{(\mathrm{D}) \mathrm{IP}}\right)$ are shown in Fig. 3. The ratios have similar trends as a function of $Z$, but the transverse Breit correction for DIPs seems to be around 1.2 times the correction in IPs while the Gaunt term giving a higher ratio. For comparison, the scaling ratio of the Gaunt correction obtained from the DIRAC code is depicted in Fig. 3 giving very similar behaviour as the corresponding correction from the GRASP code.

\section{E. Z-scaling}

In order to investigate $Z$-scaling properties of our results we have performed fitting of several computed quantities functions of $Z$. The $Z$-scaling was assumed to follow the functional form

$$
x(Z)=a Z^{n},
$$

TABLE VI. Calculated relativistic electron-electron interaction corrections (in $\mathrm{eV}$ ) in the Gaunt and frequency-independent transverse Breit formalism obtained by the GRASP code package. For comparison, values for the Gaunt correction obtained by the DIRAC code are given. For details see the text.

\begin{tabular}{lccccc}
\hline \hline & $\mathrm{Ne}$ & $\mathrm{Ar}$ & $\mathrm{Kr}$ & $\mathrm{Xe}$ & $\mathrm{Rn}$ \\
\hline Gaunt $_{\text {IP }}$, DIRAC & -0.34 & -2.45 & -23.68 & -88.86 & -418.97 \\
Gaunt $_{\text {IP }}$, GRASP & -0.34 & -2.44 & -23.53 & -88.24 & -411.42 \\
Breit $_{\text {IP }}$, GRASP & -0.33 & -2.34 & -22.14 & -82.48 & -384.46 \\
Gaunt $_{\text {DIP }}$, DIRAC & -0.41 & -3.21 & -32.21 & -123.29 & -590.13 \\
Gaunt $_{\text {DIP }}$, GRASP & -0.41 & -3.18 & -31.87 & -121.89 & -573.98 \\
Breit $_{\text {DIP }}$, GRASP & -0.39 & -2.93 & -28.83 & -109.73 & -518.26 \\
\hline
\end{tabular}

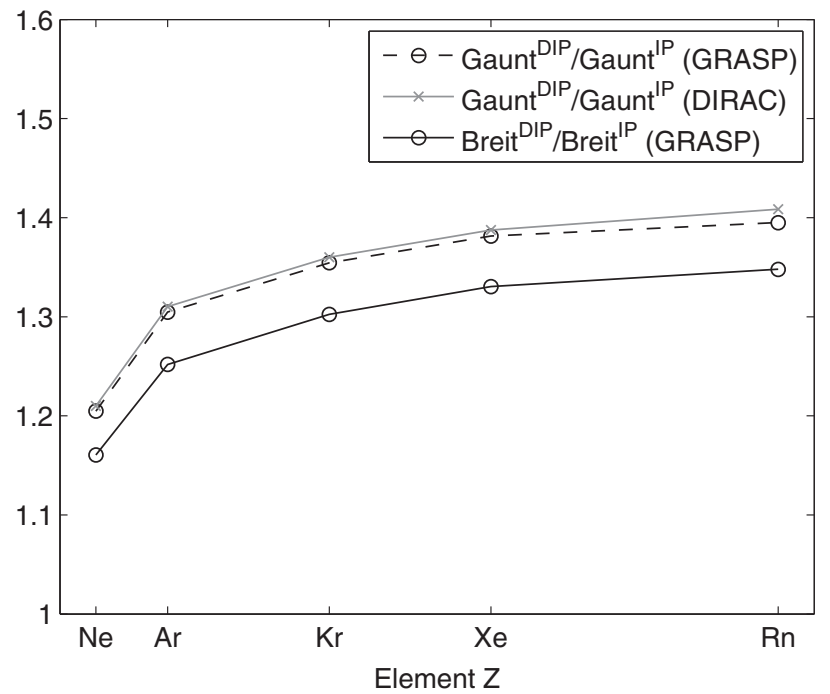

FIG. 3. The ratio of the relativistic electron-electron interaction corrections in the $1 s$ single and double ionization potentials for the noble gas series from $\mathrm{Ne}$ to $\mathrm{Rn}$. The ratio is depicted for the Gaunt and (frequency-independent) transverse Breit correction from the GRASP code package and for the Gaunt correction from the DIRAC package. For more details, see text.

where $x$ denotes the quantity to be fitted, $a$ and $n$ are the parameters to be solved. The values of the parameters are given in Table VII. From the fitting values of Table VII, the quantities related to double core ionization are seen to follow roughly a similar scaling as the ones related to the single core ionization. The scaling for the transverse Breit operator follows closely the Gaunt values.

The $Z$-scaling of nonrelativistic (D)IPs is very close to quadratic, in agreement with Moseley's law for $K$-shell x-ray

TABLE VII. Fitting parameters for $Z$-scaling of (D)IPs and relativistic contributions. For details, see the text.

\begin{tabular}{|c|c|c|}
\hline & $a$ & $n$ \\
\hline $\mathrm{IP}_{\mathrm{NR}}$ & 7.794 & 2.095 \\
\hline $\mathrm{IP}_{\mathrm{DC}}$ & 4.667 & 2.236 \\
\hline $\mathrm{IP}_{\mathrm{DCG}}$ & 4.717 & 2.233 \\
\hline Dirac $_{\text {IP }}$ & $3.399 \times 10^{-5}$ & 4.403 \\
\hline Gaunt IP & $-1.545 \times 10^{-4}$ & 3.326 \\
\hline $\mathrm{DCG}_{\mathrm{IP}}$ & $2.542 \times 10^{-5}$ & 4.459 \\
\hline $\operatorname{relax}_{\mathrm{NR}}^{\mathrm{IP}}$ & -5.931 & 0.600 \\
\hline $\operatorname{relax}_{\mathrm{DC}}^{\mathrm{IP}}$ & -4.348 & 0.701 \\
\hline $\operatorname{relax}_{\mathrm{DCG}}^{\mathrm{IP}}$ & -3.836 & 0.742 \\
\hline$\Delta_{\mathrm{DC}}^{\mathrm{IP}}$ & $-1.009 \times 10^{-4}$ & 2.670 \\
\hline$\Delta_{\mathrm{DCG}}^{\mathrm{IP}}$ & $-1.988 \times 10^{-4}$ & 2.598 \\
\hline $\mathrm{DIP}_{\mathrm{NR}}$ & 16.500 & 2.083 \\
\hline DIP $_{\mathrm{DC}}$ & 9.869 & 2.226 \\
\hline DIP $_{\text {DCG }}$ & 9.945 & 2.223 \\
\hline Dirac $_{\text {DIP }}$ & $7.269 \times 10^{-5}$ & 4.390 \\
\hline Gaunt $_{\text {DIP }}$ & $-1.869 \times 10^{-4}$ & 3.360 \\
\hline $\mathrm{DCG}_{\mathrm{DIP}}$ & $6.012 \times 10^{-5}$ & 4.427 \\
\hline $\operatorname{relax}_{\mathrm{NR}}^{\mathrm{DIP}}$ & -21.276 & 0.621 \\
\hline $\operatorname{relax}_{\mathrm{DC}}^{\mathrm{DIP}}$ & -15.638 & 0.721 \\
\hline $\operatorname{relax}_{\mathrm{DCG}}^{\mathrm{DIP}}$ & -14.088 & 0.756 \\
\hline$\Delta_{\mathrm{DC}}^{\mathrm{DIP}}$ & $-3.936 \times 10^{-4}$ & 2.668 \\
\hline$\Delta_{\mathrm{DCG}}^{\mathrm{DIP}}$ & $-6.814 \times 10^{-4}$ & 2.614 \\
\hline
\end{tabular}


line energies for light elements. ${ }^{32}$ However, at the high $Z$ end the one-electron relativistic effects in (D)IPs are seen to give rise to scaling faster than $Z^{4}$ and the two-electron effects approximately as $Z^{3.3}$. When combined, the one-electron effects dominate, as seen from Table I, and the relativistic effects are seen to scale approximately as $Z^{4.4}$ ( $\mathrm{DCG}_{(\mathrm{D}) I \mathrm{P}}$ in Table VII), obeying roughly quadratically the scaling of relativistic valence effects. ${ }^{33}$ This implies that the relativistic (D)IPs scale faster than the quadratic law, approximately as $Z^{2.2}$, which is seen from Table VII.

Orbital relaxation energies in (D)IPs show less than linear scaling in all theoretical frameworks and for both core ionization degrees. However, the trend of scaling becomes closer to linear when relativistic effects are included as seen from entries labeled as "relax" in Table VII. Finally, according to our calculations, the relaxation induced relativistic energies $(\Delta)$ in (D)IPs scale approximately as $Z^{2.6}$.

\section{CONCLUSIONS}

Motivated by ongoing experimental research on hollow atoms and multiple ionized states using modern $\mathrm{x}$-ray sources such as the Linac Coherent Light Source, we explore the capability of the state-of-art theory and software to predict the energetics of such states. For this purpose, we presented a series of calculations for $1 s$ single and double ionization energies for the noble gas series from $\mathrm{Ne}$ to $\mathrm{Rn}$. Nonrelativistic and relativistic theory, the latter encompassing the Dirac-Coulomb, Dirac-Coulomb-Gaunt, and Dirac-Coulomb-Breit Hamiltonians, were employed. Direct additivity of the relativistic corrections to the single electron to the double electron ionization potentials were explored and was found to gradually improve toward the heavy elements. The effect of the used nuclear model was found to be small. The relativistic effects contributing to the core ionization potential were found to scale approximately as $Z^{4.4}$ whereas orbital relaxation energies obey a $Z$-scaling slower than linear. The present work indicates that accurate predictions of double core hole ionization potentials is now a realistic proposition for elements across the full periodic table.

\section{ACKNOWLEDGMENTS}

J.N. would like to thank the Finnish National Graduate School in materials Physics and Magnus Ehrnrooth's foundation for financial support during the work. Docent Sami Heinäsmäki is acknowledged for his advice considering the GRASP calculations. We also thank Professor Jan-Erik Rubensson for pointing out some key references.

${ }^{1}$ J. H. D. Eland, O. Vieuxmaire, T. Kinugawa, P. Lablanquie, R. I. Hall, and F. Penent, Phys. Rev. Lett. 90, 053003 (2003).
${ }^{2}$ J. H. D. Eland, S. S.W. Ho, and H. L. Worthington, Chem. Phys. 290, 27 (2003).

${ }^{3}$ R. Santra, N. Kryzhevoi, and L. S. Cederbaum, Phys. Rev. Lett. 103, 013002 (2009).

${ }^{4}$ L. Young, E. P. Kanter, B. Krässig, Y. Li, A. M. March, S. T. Pratt, R. Santra, S. H. Southworth, N. Rohringer, L. F. DiMauro, G. Doumy, C. A. Roedig, N. Berrah, L. Fang, M. Hoener, P. H. Bucksbaum, J. P. Cryan, S. Ghimire, J. M. Glownia, D. A. Reis, J. D. Bozek, C. Bostedt, and M. Messerschmidt, Nature (London) 466, 56 (2010).

${ }^{5}$ L. Fang, M. Hoener, O. Gessner, F. Tarantelli, S. T. Pratt, O. Kornilov, C. Buth, M. Gühr, E. P. Kanter, C. Bostedt, J. D. Bozek, P. H. Bucksbaum, M. Chen, R. Coffee, J. Cryan, M. Glownia, E. Kukk, S. R. Leone, and N. Berrah, Phys. Rev. Lett. 105, 083005 (2010).

${ }^{6} \mathrm{~K}$. Hino, T. Ishihara, F. Shimizu, N. Toshima, and J. H. McGuire, Phys. Rev. A 48, 1271 (1993).

${ }^{7}$ J. Hoszowska, A. K. Kheifets, J.-Cl. Dousse, M. Berset, I. Bray, W. Cao, K. Fennane, Y. Kayser, M. Kavčič, J. Szlachetko, and M. Szlachetko, Phys. Rev. Lett. 102, 073006 (2009), and references therein.

${ }^{8}$ J. Hoszowska, A. S. Kheifets, J.-Cl. Dousse, I. Bray, W. Cao, K. Fennane, Y. Kayser, M Kavčič, J. Szlachetko, and M. Szlachetko, J. Phys.: Conf. Ser. 212, 012006 (2010).

${ }^{9}$ R. Moshammer, Y. H. Jiang, L. Foucar, A. Rudenko, Th. Ergler, C. D. Schröter, S. Lüdemann, K. Zrost, D. Fischer, J. Titze, T. Jahnke, M. Schöffler, T. Weber, R. Dörner, T. J. M. Zouros, A. Dorn, T. Ferger, K. U. Kühnel, S. Düsterer, R. Treusch, P. Radcliffe, E. Plönjes, and J. Ullrich, Phys. Rev. Lett. 98, 203001 (2007).

${ }^{10}$ A. Rudenko, L. Foucar, M. Kurka, Th. Ergler, K. U. Kühnel, Y. H. Jiang, A. Voitkiv, B. Najjari, A. Kheifets, S. Lüdemann, T. Havermeier, M. Smolarski, S. Schössler, K. Cole, M. Schöffler, R. Dörner, S. Düsterer, W. Li, B. Keitel, R. Treusch, M. Gensch, C. D. Schröter, R. Moshammer, and J. Ullrich, Phys. Rev. Lett 101, 073003 (2008).

${ }^{11}$ H. Ågren and H. J. Aa. Jensen, Chem. Phys. 172, 45 (1993).

${ }^{12}$ H. Ågren, J. Nordgren, L. Selander, C. Nordling, and K. Siegbahn, Phys. Scr. 18, 499 (1978).

${ }^{13}$ P. Pelicon, I. Čadež, M. Žitnik, Ž. Šmit, S. Dolenc, A. Mühleisen, and R. I. Hall, Phys. Rev. A 62, 022704 (2000).

${ }^{14}$ S. H. Southworth, E. P. Kanter, B. Krässig, and L. Young, Phys. Rev. A 67, 062712 (2003).

${ }^{15}$ E. P. Kanter, I. Ahmad, R. W. Dunford, D. S. Gemmell, B. Krässig, S. H. Southworth, and L. Young, Phys. Rev. A 73, 022708 (2006).

${ }^{16}$ J. A. Bearden and A. F. Burr, Rev. Mod. Phys. 39, 125 (1967).

${ }^{17}$ L. Pettersson, J. Nordgren, L. Selander, C. Nordling, K. Siegbahn, and Hans Ågren, J. Electron Spectrosc. Relat. Phenom. 27, 29 (1982).

${ }^{18} \mathrm{H}$. Winter and F. Aumayr, J. Phys. B 32, R39 (1999).

${ }^{19}$ M. H. Chen, Phys. Rev. A 44, 239 (1991).

${ }^{20} \mathrm{See} \quad$ https://slacportal.slac.stanford.edu/sites/lcls_public/Pages/Default. aspx for the Linac Coherent Light Source homepage.

${ }^{21}$ J.-M. Lévy-Leblond, Commun. Math. Phys 6, 286 (1967).

${ }^{22}$ DIRAC, a relativistic $a b$ initio electronic structure program, Release DIRAC08.beta, written by L. Visscher, H. J. Aa. Jensen, T. Saue et al.

${ }^{23}$ K. G. Dyall, I. P. Grant, C. T. Johnson, F. A. Parpia, and E. P. Blummer, Comput. Phys. Comm. 55, 425 (1989).

${ }^{24}$ S. Fritzsche, J. Electron Spectrosc. Relat. Phenom. 114-116, 1155 (2001).

${ }^{25}$ I. P. Grant, Relativistic Quantum Theory of Atoms and Molecules (Springer, New York, 2007).

${ }^{26}$ T. H. Dunning, Jr., J. Chem. Phys. 90, 1007 (1989).

${ }^{27}$ D. E. Woon and T. H. Dunning, Jr., J. Chem. Phys. 98, 1358 (1993).

${ }^{28}$ K. G. Dyall, Theor. Chem. Acc. 115, 441 (2006).

${ }^{29}$ K. G. Dyall, Theor. Chem. Acc. 108, 335 (2002).

${ }^{30}$ L. Visscher, K. G. Dyall, At. Data Nucl. Data Tables 67, 207 (1997).

${ }^{31}$ L. C. Snyder, J. Chem. Phys. 55, 95 (1971).

${ }^{32}$ H. G. J. Moseley, Philos. Mag. 26, 1024 (1913); H. G. J. Moseley, Philos. Mag. 27, 703 (1914).

${ }^{33}$ P. Pyykkö, Chem. Rev. 88, 563 (1988). 